\title{
Improving the Quality of QUS Imaging Using Full Angular Spatial Compounding
}

\author{
Roberto J. Lavarello, Jose R. Sanchez, and Michael L. Oelze \\ Bioacoustics Research Laboratory \\ Department of Electrical and Computer Engineering \\ University of Illinois at Urbana-Champaign \\ Urbana, IL 61801 \\ Email: lavarell@illinois.edu
}

\begin{abstract}
Quantitative ultrasound (QUS) imaging techniques make use of information from backscattered echoes discarded in conventional B-mode imaging. Using scattering models and spectral fit methods, properties of tissue microstructure can be estimated. The variance of QUS estimates is usually reduced by processing data obtained from a region of interest (ROI) whose dimensions are larger than the resolution cell of B-mode imaging, which limits the spatial resolution of the technique. In this work, the use of full angular (i.e., $360^{\circ}$ ) spatial compounding is proposed to extend the trade-off between estimate variance and spatial resolution of QUS. Simulations were performed using an f/4, 10-MHz transducer with 50\% -6-dB bandwidth and a synthetic phantom consisting of two eccentric circular cylindrical regions. The inner and outer cylinders had radii of $7 \mathrm{~mm}$ and $12.5 \mathrm{~mm}$, respectively, and nine scatterers per resolution cell. The average scatterer diameters (ASDs) for the outer and inner cylinders were $50 \mu \mathrm{m}$ and $25 \mu \mathrm{m}$, respectively. ASD estimates were obtained using radio frequency data at up to 128 angles of view. When using ROIs of size $16 \lambda$ by $16 \lambda$, the use of multiple view data reduced the ASD standard deviations in the outer and inner cylinders from 7.4 $\mu \mathrm{m}$ and $14.4 \mu \mathrm{m}$ to $1.5 \mu \mathrm{m}$ and 2.5 $\mu \mathrm{m}$, respectively. When using ROIs of size $8 \lambda$ by $8 \lambda$, the use of multiple view data reduced the ASD standard deviations in the outer and inner cylinders from $13.7 \mu \mathrm{m}$ and $19.6 \mu \mathrm{m}$ to $2.5 \mu \mathrm{m}$ and $3.7 \mu \mathrm{m}$, respectively. Experimental validation was obtained using a $10 \mathrm{MHz}, \mathrm{f} / 4$ transducer to analyze a $2 \mathrm{~cm}$ diameter homogeneous agar phantom with embedded glass spheres of diameters between $45 \mu \mathrm{m}$ and $53 \mu \mathrm{m}$. When using ROIs of size $10 \lambda$ by $10 \lambda$ and 32 angles of view, the ASD standard deviation was reduced from $24.6 \mu \mathrm{m}$ to $4.8 \mu \mathrm{m}$. This value was below 10.4 $\mu \mathrm{m}$, the ASD standard deviation obtained using single view data and ROIs of size $20 \lambda$ by $20 \lambda$. Therefore, the use of full angular compounding was found to significantly improve the trade-off between spatial resolution in QUS imaging and precision of QUS estimates. These results suggest that QUS imaging can achieve optimal performance on a platform capable of producing views of an object from $360^{\circ}$, e.g., a tomographic breast scanner.
\end{abstract}

\section{INTRODUCTION}

The quantitative ultrasound (QUS) imaging technique based on ultrasonic backscatter has proven potential for tissue characterization. Experimental work in the literature includes explorations of ocular lesions [1], prostate [2], kidney [3], and liver [4], among others. If multiple scattering is neglected, backscattered spectrum measurements can be fitted to a parametric model that represents how a single scatterer radiates sound as a function of frequency. In practice, coherent scattering terms are also present in the measured spectra.
Therefore, the spectrum is not completely predicted by the single scatterer model and variations are introduced in the QUS estimates. The variance of the estimates can be reduced by averaging the spectra of adjacent, partially uncorrelated Alines at the expense of spatial resolution.

Coherent scattering is responsible for the speckle pattern common to ultrasound B-mode images. The speckle pattern created by a distribution of scatterers changes with its position relative to the ultrasonic transducer [5]. This fact has been exploited for several years to reduce speckle variance through the use of spatial compounding [6]. In this work, the use of angular compounding to increase the number of independent backscattered measurements for QUS estimation without degrading the spatial resolution was explored. Related studies can be found in the literature using the limited compounding capabilities that can be obtained with linear arrays [7], [8]. The work presented here extends those results to deal with the case in which full angular coverage of the imaging target can be obtained, such as in breast imaging. Even further, this works explores how the variance reduction provided by compounding can be traded off for improving the spatial resolution of QUS images.

\section{Methods}

\section{A. Quantitative ultrasound (QUS) imaging}

For completeness, the QUS estimation process is described here. The radio frequency (rf) data collected by ultrasonic transducers is usually processed for image formation by displaying B-mode images, i.e., grayscale images where the brightness of each pixel is proportional to the amplitude of the log compressed envelope of the ultrasonic echoes. QUS estimates are calculated from measurements of backscattering coefficients, which are also obtained from the rf data. Backscattering coefficients can be obtained at different depths per A-line by calculating the magnitude of the power spectrum of the rf data after proper gating with a windowing function. The length of the window defines the axial resolution of the QUS images. The same process is repeated for several adjacent scan lines and the resulting spectra are averaged together in order to reduce the coherent scattering component. The number of scan lines that are averaged determines the lateral resolution of QUS images. The box limited by the length 
of the range gate and the number of adjacent scan lines is termed the region of interest (ROI). In practice, minimum ROI dimensions of 15 pulse lengths axially and 4 beamwidths laterally have been suggested as a good compromise between spatial resolution and estimate accuracy [9].

In order to remove the dependence on the imaging system properties, the backscattered spectrum is divided by a calibration spectrum. Neglecting attenuation, the normalized power spectrum $S(f)$ of the measured rf data can be expressed as [10]

$$
S(k)=S_{\xi}(2 k) * S_{D}(2 k) * S_{G}(2 k),
$$

where $S_{\xi}(k)$ is the Fourier transform of the spatial autocorrelation function (ACF) describing the acoustic impedance of the underlying tissue microstructure, $S_{D}(k)$ is the Fourier transform of the ACF of the transducer beam's directivity function, and $S_{G}(k)$ is the Fourier transform of the AFC of the gating function in the axial direction. For weakly focused transducers and long gate lengths, the frequency variations of $S(k)$ depend only on $S_{\xi}(k)$. Typically, scattering is assumed to be caused by an ensemble of radially symmetric structures such as fluid Gaussian or solid spheres [11]. Using these models, estimates of the average scatterer diameter (ASD) and the acoustic concentration are used to describe tissue microstructure.

\section{B. Full angular compounding}

Spatial compounding was designed as a tool for reducing the speckle and therefore improving the contrast in B-mode images. The principle of spatial compounding is that the speckle pattern changes with the relative position between the transducer and the scattering region. Therefore, if the same region is observed with a transducer at different positions and/or orientations, the envelopes of the received echoes can be averaged in order to reduce the variance of the resulting image. Most ultrasonic images are constructed using linear or phased arrays, which limits the amount of transducer position variation that can be obtained.

Full angular compounding capabilities, which can be obtained with acoustic tomography scanners, can also be used to improve on the quality of QUS images. The approach proposed here consists of obtaining rf data from each ROI at different illumination angles, as shown in Fig. 1. With this approach, more $\mathrm{rf}$ measurements corresponding to the same region of space can be obtained without changing the size of the ROI. Therefore, the variance of the QUS estimates can be reduced without degrading the spatial resolution.

\section{Simulation Results}

Simulated data was generated using Gaussian scatterers (effective ASD $=50 \mu \mathrm{m}$ ) distributed on a cylindrical region with a radius of $12.5 \mathrm{~mm}$. A total of nine scatterers per resolution cell were randomly distributed in the simulated volume. The simulated $\mathrm{f} / 4$ transducer had a focal distance of $45 \mathrm{~mm}$. The transmitted pulse had a Gaussian spectrum with $50 \%-6-\mathrm{dB}$ bandwidth centered at $10 \mathrm{MHz}$. A least squares
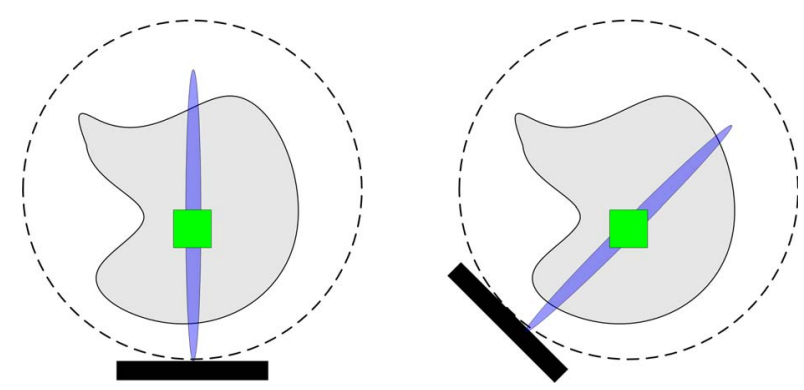

Fig. 1. Propose full angular compounding configuration. The aperture (black rectangle) is rotated around the imaging target (irregular shape) over a full circular trajectory. The figure shows an ROI (green square) illuminated at 0 (left) and 45 (right) degrees.

linear estimator applied to the compensated backscattered spectrum was used to obtain the value of the ASD for each ROI [12]. Estimates were obtained by using data simulated at $N_{a}=1,2,4, \ldots 128$ angles of view uniformly distributed between 0 and $360^{\circ}$.

\begin{tabular}{|c|c|c|c|c|c|c|c|c|}
\hline \multirow{2}{*}{$\begin{array}{c}\text { ROI } \\
\text { size }\end{array}$} & 1 & 2 & 4 & 8 & 16 & 32 & 64 & 128 \\
\hline $\begin{array}{c}16 \lambda \times \\
16 \lambda\end{array}$ & 7.2 & 6.6 & 4.7 & 3.2 & 2.1 & 1.5 & 1.2 & 1.2 \\
\hline $\begin{array}{c}8 \lambda \times \\
8 \lambda\end{array}$ & 13.8 & 11.6 & 7.9 & 5.6 & 3.7 & 2.5 & 2.1 & 2.0 \\
\hline
\end{tabular}

TABLE I

STANDARD DEVIATION OF THE ASD ESTIMATES WHEN USING FULL

ANGULAR COMPOUNDING AND A HOMOGENEOUS PHANTOM WITH GAUSSIAN SCATTERERS. THE TRUE EFFECTIVE SCATTERER DIAMETER WAS SET TO $50 \mu \mathrm{M}$.

As expected, the use of multiple view data improved on the precision of ASD estimates as quantified by the reduction in the standard deviation. For most of the cases, doubling the number of views reduced the standard deviation close to a factor of $\sqrt{2}$. However, it can be noticed that the standard deviation did not significantly change between the $N_{a}=1$ and $N_{a}=2$ cases, and the $N_{a}=64$ and $N_{a}=128$ cases. The degree of correlation in the backscattered coefficient measurements was indirectly measured by quantifying the reduction in ASD standard deviation when combining data collected at two angles of view. The results are shown graphically on Fig. 2 when using the homogeneous phantom with $50 \mu \mathrm{m}$ scatterers. The correlation length around $0^{\circ}$ was approximately $5^{\circ}$. The correlation peak around $180^{\circ}$ occurred due to the symmetry of the transmitted pulse.

The advantages of variance reduction are clearer when inhomogeneous imaging targets are analyzed. A simulated phantom consisting of two cylindrical circular regions was also analyzed using QUS and compounding. The inner and outer cylinders had radii of $7 \mathrm{~mm}$ and $12.5 \mathrm{~mm}$, respectively, and nine scatterers per resolution cell. The effective ASD in the outer and inner regions were $50 \mu \mathrm{m}$ and $25 \mu \mathrm{m}$, respectively. 


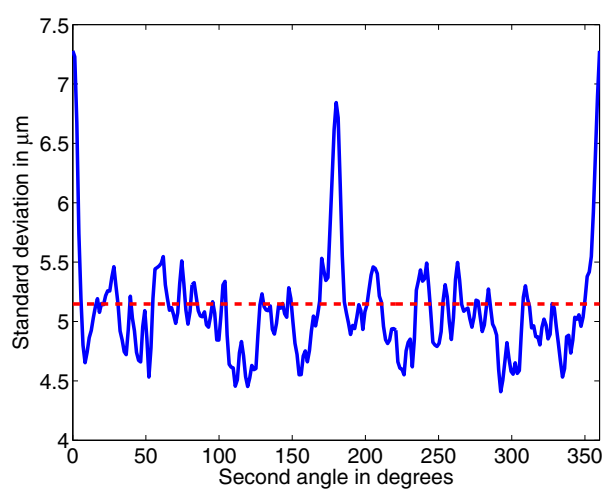

Fig. 2. ASD standard deviation when using two angles of view as a function of the angular separation between views. The plot shows the standard deviation for angular separations between 0 and 360 degrees. Both the standard deviation (blue line) and the ideal $1 / \sqrt{2}$ decay (red line) are shown.

A total of 64 angles of view were used in the estimation. When using ROIs of size $16 \lambda$ by $16 \lambda$, the use of multiple view data reduced the ASD standard deviations in the outer and inner cylinders from $7.4 \mu \mathrm{m}$ and $14.4 \mu \mathrm{m}$ to $1.5 \mu \mathrm{m}$ and $2.5 \mu \mathrm{m}$, respectively. When using ROIs of size $8 \lambda$ by $8 \lambda$ (as shown shown in Fig. 3), the use of multiple view data reduced the ASD standard deviations in the outer and inner cylinders from $13.7 \mu \mathrm{m}$ and $19.6 \mu \mathrm{m}$ to $2.5 \mu \mathrm{m}$ and $3.7 \mu \mathrm{m}$, respectively.

\section{EXPERIMENTAL RESULTS}

Experimental results were obtained using a $10 \mathrm{MHz}, \mathrm{f} / 4$ transducer focused at a depth of 2 inches. The phantom was a homogeneous $1 \mathrm{~cm}$ radius cylindrical phantom of $4 \%$ by weight agar with 2 grams per liter of 45 to $53 \mu \mathrm{m}$ diameter glass beads. The attenuation coefficient was estimated to be $0.1 \mathrm{~dB} / \mathrm{cm} / \mathrm{MHz}$. Rf data was collected between $0^{\circ}$ and $360^{\circ}$ in increments of $11.25^{\circ}$ for a total of 32 data sets. The estimation was performed using the $-6-\mathrm{dB}$ bandwidth of the transducer, the exact scattering model for a solid sphere [13], and point attenuation compensation [11]. ASD estimates were obtained using ROI sizes of $3 \mathrm{~mm}$ by $3 \mathrm{~mm}(20 \lambda$ by $20 \lambda$ ), and $1.5 \mathrm{~mm}$ by $1.5 \mathrm{~mm}(10 \lambda$ by $10 \lambda)$. The ASD statistics without compounding were calculated considering all 32 sets of QUS estimates corresponding to each incidence angle. Bmode images with a parametric image overlay representing the ASD estimates are shown in Figs. 4 and 5.

When using the larger ROI size, the ASD mean and standard deviation values were $53.8 \mu \mathrm{m}$ and $10.4 \mu \mathrm{m}$ without compounding, and $53.9 \mu \mathrm{m}$ and $2.5 \mu \mathrm{m}$ with compounding. The use of compounding resulted in a reduction in the standard deviation by a factor of 4.2, whereas an optimum factor of $\sqrt{32}$ was expected. This apparent limitation in standard deviation reduction can be due to the variation of the glass sphere diameters $(49+/-4 \mu \mathrm{m})$. When using the smaller ROI size, the ASD mean and standard deviation values were $62.4 \mu \mathrm{m}$ and $24.6 \mu \mathrm{m}$ without compounding, and $62.7 \mu \mathrm{m}$ and $4.8 \mu \mathrm{m}$ with compounding. The improvement factor in this case was 5.1 , closer to the optimum value of $\sqrt{32}$. Furthermore, the standard deviation was smaller than the one obtained in the single view case using the larger $20 \lambda$ by $20 \lambda$ ROI size.

\section{CONCLUSIONS}

Both through simulations and experiments, the use of full angular compounding was found to significantly improve the trade off between spatial resolution in QUS imaging and precision of QUS estimates. These results suggest that QUS imaging can achieve optimal performance on a platform capable of producing views of an object from $360^{\circ}$, e.g., a tomographic breast scanner.

\section{ACKNOWLEDGEMENTS}

The authors would like to thank Andrew Battles and Dr. William D. O'Brien, Jr. This work was funded by a grant from the National Institutes of Health (R43 CA12152).

\section{REFERENCES}

[1] E.J. Feleppa, F.L. Lizzi, D.J. Coleman, and M.M. Yaremko, "Diagnostic spectrum analysis in ophthalmology: a physical perspective," Ultrasound in Medicine and Biology, vol. 12, no. 8, pp. 621-631, August 1986.

[2] E.J. Feleppa, A. Kalisz, J.B. Sokil-Melgar, F.L. Lizzi, T. Liu, A.L. Rosado, M.C. Shao, W.R. Fair, Y. Wang; M.S. Cookson, V.E. Reuter, and W.D.W Heston, "Typing of prostate tissue by ultrasonic spectrum analysis," IEEE Transactions on Ultrasonics, Ferroelectrics, and Frequency Control, vol. 43, no. 4, pp. 609-619, July 1996.

[3] M.F. Insana, T.J. Hall, and J.L. Fishback, "Identifying acoustic scattering sources in normal renal parenchyma from the anisotropy in acoustic properties," Ultrasound in Medicine and Biology, vol. 17, no. 6, pp. 613-626, 1991.

[4] F.L. Lizzi, D.L. King, M.C. Rorke, J. Hui, M. Ostromogilsky, M.M Yaremko, E.J. Feleppa, and P. Wai, "Comparison of theoretical scattering results and ultrasonic data from clinical liver examinations," Ultrasound in Medicine and Biology, vol. 14, no. 5, pp. 377-385, 1988.

[5] C.B. Buckhardt, "Speckle in ultrasound B-mode scans," IEEE Transactions on Sonics and Ultrasonics, vol. SU-25, no. 1, pp. 1-6, January 1978.

[6] G.E. Trahey, S.W. Smith, and O.T von Ramm, "Speckle pattern correlation with lateral aperture translation: Experimental results and implications for spatial compounding," IEEE Transactions on Ultrasonics, Ferroelectrics, and Frequency Control, vol. 33, no. 3, pp. 257-264, May 1986.

[7] A.L. Gerig, T. Varghese, and J.A. Zagzebsky, "Improved parametric imaging of scatterer size estimates using angular compounding," IEEE Transactions on Ultrasonics, Ferroelectrics, and Frequency Control, vol. 51, no. 6, pp. 708-715, June 2004.

[8] A.L. Gerig, Q. Chen, J.A. Zagzebsky, and T. Varghese, "Correlation of ultrasonic scatterer size estimates for the statistical analysis and optimization of angular compounding," Journal of the Acoustical Society of America, vol. 116, no. 3, pp. 1832-1841, September 2004.

[9] M. Oelze and W.D. O'Brien, Jr., "Defining optimal axial and lateral resolution for estimating scatterer properties from volumes using ultrasound backscatter,' Journal of the Acoustical Society of America, vol. 115, no. 6, pp. 3226-3234, June 2004.

[10] F.L. Lizzi, M. Astor, T. Liu, C. Deng, D.J. Coleman, and R.H. Silverman, "Ultrasonic spectrum analysis for tissue assays and therapy evaluation," International Journal of Imaging Systems and Technology, vol. 8, no. 1, pp. 3-10, 1997.

[11] M.F. Insana, R.F. Wagner, D.G. Brown, and T.J. Hall, "Describing smallscale structure in random media using pulse-echo ultrasound," Journal of the Acoustical Society of America, vol. 87, no. 1, pp. 179-192, January 1990.

[12] M.L. Oelze, J.F. Zachary, and W.D. O'Brien, Jr., "Characterization of tissue microstructure using ultrasonic backscatter: Theory and technique for optimization using a Gaussian form factor,' Journal of the Acoustical Society of America, vol. 112, no. 3, pp. 1202-1211, September 2002.

[13] R. Hickling, "Analysis of echoes from a solid elastic sphere in water," Journal of the Acoustical Society of America, vol. 34, no. 10, pp. 15821562, October 1962. 

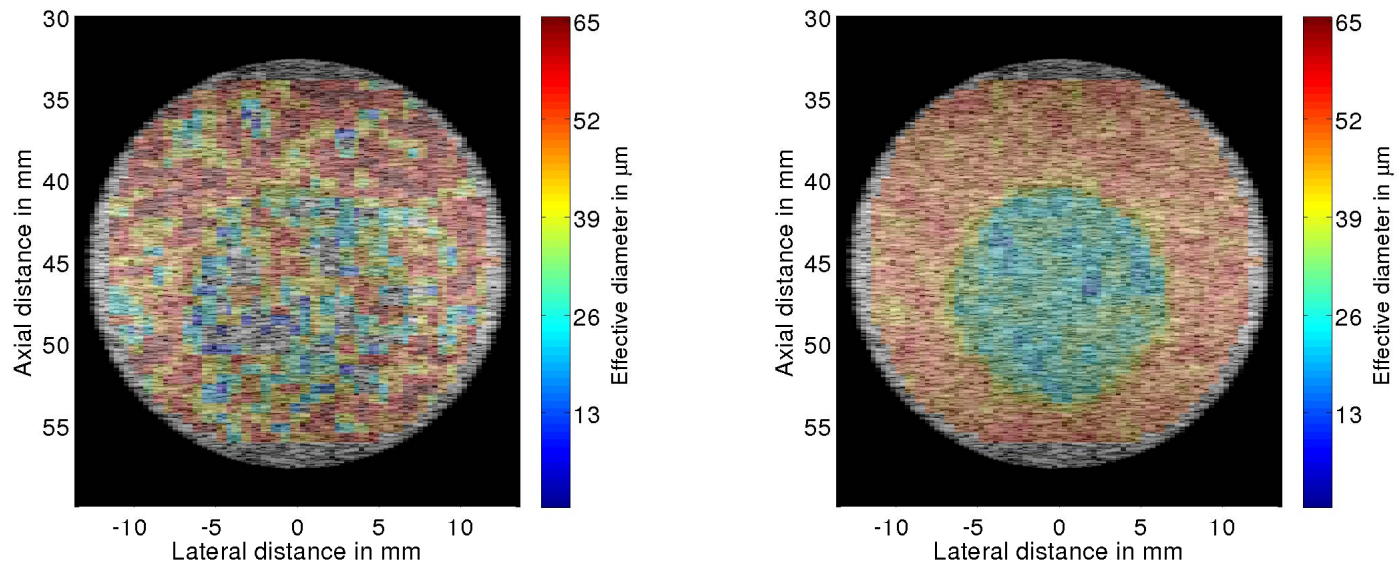

Fig. 3. ASD estimates from a simulated phantom consisting of two cylindrical regions, using ROIs of size of $1.2 \mathrm{~mm}$ by $1.2 \mathrm{~mm}(8 \lambda$ by $8 \lambda)$. The ASD estimates were obtained using data from one (left) and 64 (right) angles of view.
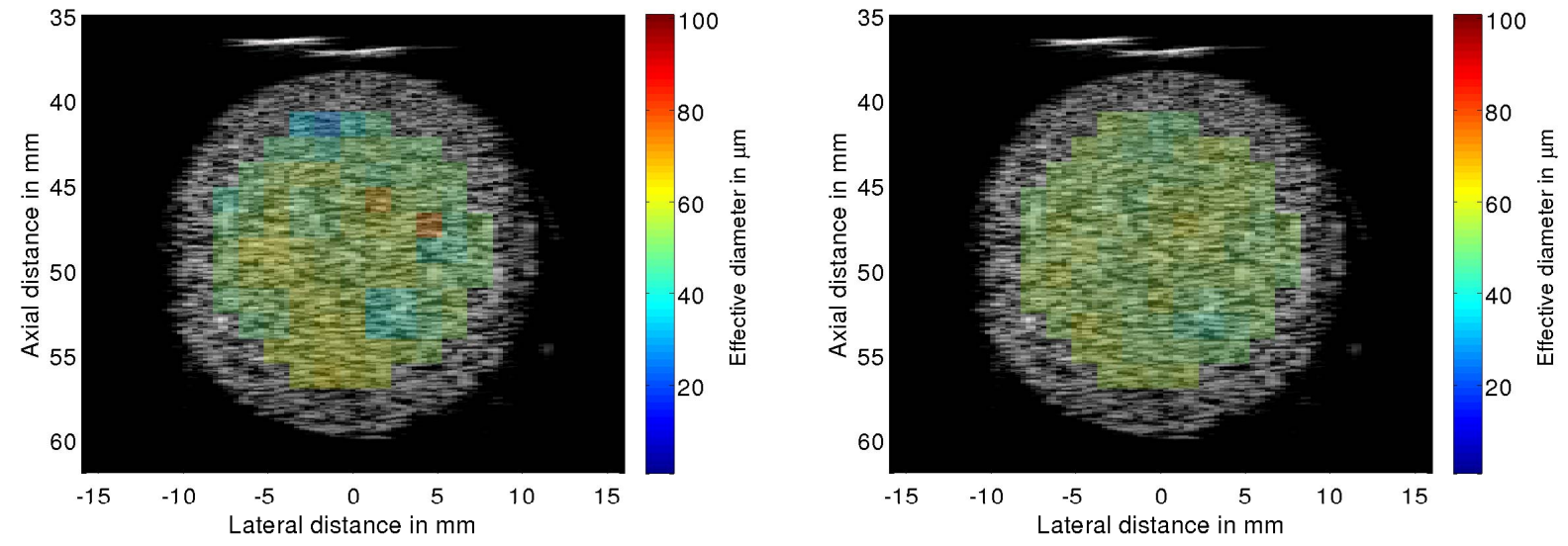

Fig. 4. ASD estimates from experimental measurements of an agar phantom, using ROIs of size $3 \mathrm{~mm}$ by $3 \mathrm{~mm}(20 \lambda$ by $20 \lambda)$. The ASD estimates were obtained using data from one (left) and 32 (right) angles of view.
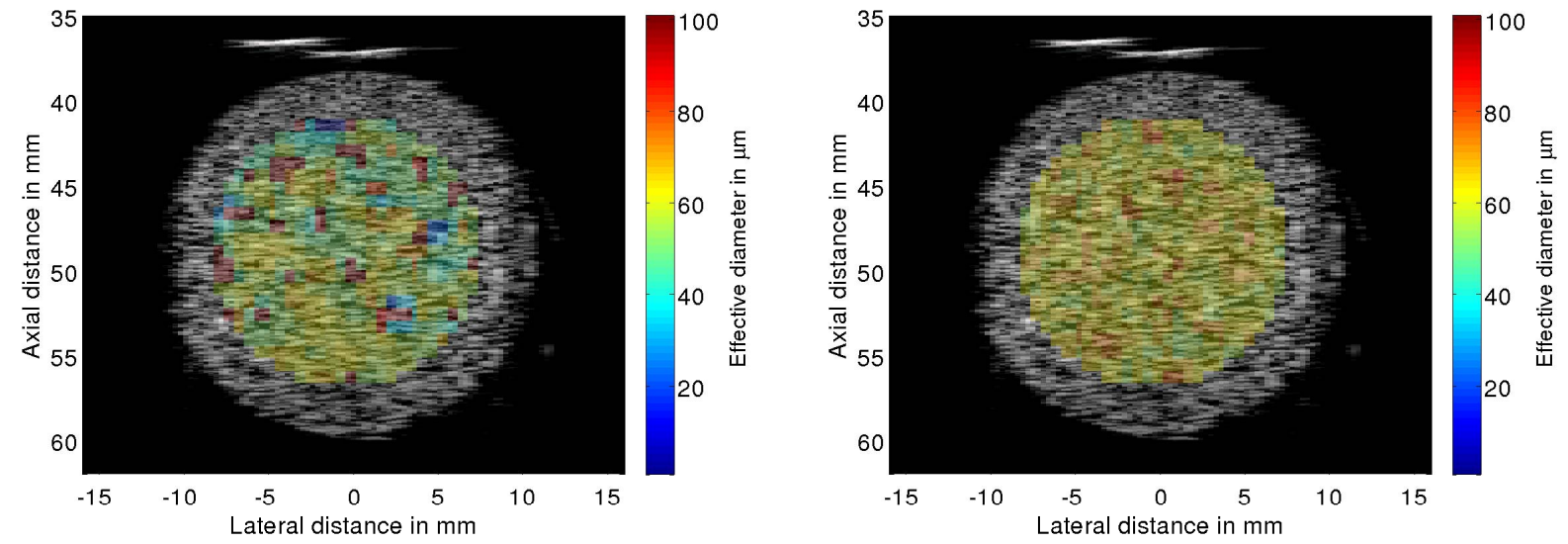

Fig. 5. ASD estimates from experimental measurements of an agar phantom, using ROIs of size $1.5 \mathrm{~mm}$ by $1.5 \mathrm{~mm}(10 \lambda$ by $10 \lambda)$. The ASD estimates were obtained using data from one (left) and 32 (right) angles of view. 